\title{
Sost Deficiency does not Alter Bone's Lacunar or Vascular Porosity in Mice
}

\author{
Henry Mosey ${ }^{1}$, Juan A. Núñez ${ }^{2}$, Alice Goring ${ }^{2}$, Claire E. Clarkin ${ }^{2}$, Katherine A. Staines ${ }^{3}$, \\ Peter D. Lee ${ }^{4}$, Andrew A. Pitsillides ${ }^{1}$ and Behzad Javaheri ${ }^{1 *}$ \\ 'Skeletal Biology Group, Comparative Biomedical Sciences, The Royal Veterinary College, London, United Kingdom, \\ ${ }^{2}$ Faculty of Natural and Environmental Sciences, Biological Sciences, University of Southampton, Southampton, \\ United Kingdom, ${ }^{3}$ School of Applied Sciences, Edinburgh Napier University, Edinburgh, United Kingdom, \\ ${ }^{4}$ Manchester X-Ray Imaging Facility, University of Manchester, Manchester, United Kingdom
}

SCLEROSTIN (Sost) is expressed predominantly in osteocytes acting as a negative regulator of bone formation. In humans, mutations in the SOST gene lead to skeletal overgrowth and increased bone mineral density, suggesting that SCLEROSTIN is a key regulator of bone mass. The function of SCLEROSTIN as an inhibitor of bone formation is further supported by Sost knockout $(\mathrm{KO})$ mice which display a high bone mass with

OPEN ACCESS

Edited by:

Gianluca Tozzi,

University of Portsmouth,

United Kingdom

Reviewed by:

Egon Perilli,

Flinders University, Australia

Himadri Shikhar Gupta,

Queen Mary University of London,

United Kingdom

*Correspondence:

Behzad Javaheri

bjavaheri@rvc.ac.uk

Specialty section:

This article was submitted to Mechanics of Materials,

a section of the journal

Frontiers in Materials

Received: 17 June 2017

Accepted: 28 August 2017

Published: 13 September 2017

Citation:

Mosey $H$, Núñez JA, Goring A, Clarkin CE, Staines KA, Lee $P D$,

Pitsillides AA and Javaheri B (2017)

Sost Deficiency does not Alter Bone's

Lacunar or Vascular

Porosity in Mice.

Front. Mater. 4:27.

doi: 10.3389/fmats.2017.00027 elevated bone formation. Previous studies have indicated that Sost exerts its effect on bone formation through Wnt-mediated regulation of osteoblast differentiation, proliferation, and activity. Recent in vitro studies have also suggested that SCLEROSTIN regulates angiogenesis and osteoblast-to-osteocyte transition. Despite this wealth of knowledge of the mechanisms responsible for SCLEROSTIN action, no previous studies have examined whether SCLEROSTIN regulates osteocyte and vascular configuration in cortices of mouse tibia. Herein, we image tibiae from Sost $\mathrm{KO}$ mice and their wild-type (WT) counterparts with high-resolution CT to examine whether lack of SCLEROSTIN influences the morphometric properties of lacunae and vascular canal porosity relating to osteocytes and vessels within cortical bone. Male Sost KO and WT mice ( $n=6$ /group) were sacrificed at 12 weeks of age. Fixed tibiae were analyzed using microCT to examine cortical bone mass and architecture. Then, samples were imaged by using benchtop and synchrotron nano-computed tomography at the tibiofibular junction. Our data, consistent with previous studies show that, Sost deficiency leads to significant enhancement of bone mass by cortical thickening and bigger cross-sectional area and we find that this occurs without modifications of tibial ellipticity, a measure of bone shape. In addition, our data show that there are no significant differences in any lacunar or vascular morphometric or geometric parameters between Sost KO mouse tibia and WT counterparts. We, therefore, conclude that the significant increases in bone mass induced by Sost deficiency are not accompanied by any significant modification in the density, organization, or shape of osteocyte lacunae or vascular content within the cortical bone. These data may imply that SCLEROSTIN does not modify the frequency of osteocytogenic recruitment of osteoblasts to initiate terminal osteocytic differentiation in mice.

Keywords: Sost, osteocyte, vascular porosity, microCT, lacunar porosity 


\section{INTRODUCTION}

Bone is a metabolically active tissue constantly adapting its structure to external mechanical stimuli, leading to changes in mass (Raab et al., 1991; Bennell et al., 2002), shape (Rubin, 1984), strength (Järvinen et al., 2003; Leppänen et al., 2008), and length (Howell, 1917; Steinberg and Trueta, 1981). The exact mechanisms for transformation of these mechanical signals into biological responses are not fully elucidated. Osteocytes, the most abundant cells within skeleton, are derived by a process termed osteocytogenesis from bone-forming osteoblasts. They reside within irregularly shaped ellipsoidal lacunar spaces (FranzOdendaal et al., 2006) and are reported to act as strain sensors and transducers (Bonewald and Johnson, 2008; Javaheri et al., 2014). There have been a number of hypotheses put forward as to how osteocytes achieve their mechanosensory role. One, the fluid flow hypothesis, states that mechanical loading perturbs bone fluid through the lacunar-canaliculi network producing shear forces on the osteocyte cell processes (Burger and KleinNulend, 1999; Han et al., 2004). An alternative proposes that osteocytes are vital for efficient bone remodeling and repair of bone microdamage (Verborgt et al., 2000; Ma et al., 2008). Besides these roles, osteocytes are also reported to regulate matrix mineral homeostasis (Qing and Bonewald, 2009) through osteocytic osteolysis (Bélanger, 1969; Kerschnitzki et al., 2013).

The cellular mechanisms that regulate mechanotransduction are not fully understood, but several key pathways have been identified in osteocytes. One such pathway involves control of the canonical Wnt signaling pathway by its negative regulator (Balemans et al., 2005; Weivoda et al., 2017) SCLEROSTIN; a protein encoded by the Sost gene expressed predominantly by deeply embedded mature osteocytes (Balemans et al., 2001).

SCLEROSTIN and the canonical Wnt signaling pathway regulate bone mass through several mechanisms, including stem cell renewal (Reya and Clevers, 2005), stimulation of pre-osteoblast differentiation and proliferation (Kato et al., 2002), enhancement of osteoblast activity (Kato et al., 2002; Bodine et al., 2004), inhibition of osteoblast and osteocyte apoptosis (Babij et al., 2003), regulation of osteoclastogenesis (Glass et al., 2005; Holmen et al., 2005), and modulation of adaptive response to mechanical strain (Armstrong et al., 2007; Javaheri et al., 2014). In addition, previous in vitro studies have reported that SCLEROSTIN promotes osteocytogenesis, or osteoblast-to-osteocyte differentiation (Atkins et al., 2011), and angiogenesis in human endothelial cells in vitro (Oranger et al., 2017). Moreover, Sost deficiency leads to higher matrix mineralization and enhanced bone mass; mineralization has been reported to influence terminal osteoblast differentiation into osteocytes (Cameron et al., 1967; Prideaux et al., 2012) and angiogenesis (Choi et al., 2000; Van Wesenbeeck et al., 2002). It remains unclear whether the role of SCLEROSTIN as a negative regulator of bone mass extends, however, to controlling osteocyte lacunar organization and vascular content in bone.

Visualization of osteocytes and vascular canals is difficult in bone's highly mineralized matrix. Thus, spatial characteristics, including density, volume, and shape of osteocyte lacunae and vascular porosity have, therefore, often been used as a proxy (Qiu et al., 2003; Carter et al., 2013; Javaheri et al., 2015). This is predominantly due to the fact that $3 \mathrm{D}$ quantification of osteocyte and vessel density and morphology is problematic using traditional imaging techniques including confocal microscopy. More recently, nano-computed tomography (nanoCT) has been employed to attain sub-micron resolution of lacuna and vascular porosity using benchtop CT scanners that offer submicron scanning. We (Javaheri et al., 2015) and others (Robling and Turner, 2002; Cooper et al., 2004; Mullender et al., 2005; Skedros et al., 2005; Matsumoto et al., 2006; Vatsa et al., 2008; Schneider et al., 2009, 2010; Palacio-Mancheno et al., 2014) have previously reported that nanoCT is a useful tool to quantify osteocyte and vascular porosity in 3D. However, the gold standard for such imaging remains synchrotron-based nanoCT which provides a higher X-ray flux and lower beam hardening effect due to monochromatic beam, which in principle can improve the image quality (Pacureanu et al., 2012; Carter et al., 2013; Dong et al., 2013; Kerschnitzki et al., 2013; Mader et al., 2013; Webster et al., 2013).

To our knowledge, no previous study has investigated SCLEROSTIN's role in the 3D organization of the osteocyte and vascular networks in bone. We hypothesize that Sost deficiency results in altered osteoblast-to-osteocyte transition and vascular network formation as reflected by changes in lacunar properties including numbers, volume, diameter, and shape as well as vascular parameters, respectively. To address this, we employ desktop as well as synchrotron-based nanoCT analyses of lacunar and vascular contents in a Sost-deficient mouse model.

\section{MATERIALS AND METHODS}

\section{Animal Model}

Frozen sperm from a male Sost knockout (KO) mouse was purchased from the Knockout Mouse Project Repository at the University of California Davis, CA, USA. Imported sperm used to fertilize ova from C57BL/6 wild-type (WT) mice. To obtain offspring, fertilized egg was implanted into pseudopregnant female C57BL/6 mice. Offspring were bred through several generations to obtain Sost homozygous mice on a C57BL/6 background.

Mice were provided with standard mouse chow and water ad libitum throughout the study and housed up to four per cage in polypropylene cages with wood chip and paper bedding. Weaners up to 8 weeks of age were fed a standard rodent breeding diet and thereafter a standard rodent maintenance diet (Special Diet Services, South Witham, UK). All procedures performed were reviewed and approved by the ethics committee of the Royal Veterinary College (London, UK) and complied with the UK Animals (Scientific Procedures) Act 1986.

\section{Imaging High-Resolution Micro and NanoCT MicroCT}

Two groups of mice, male Sost KO and WT mice ( $n=6 /$ group), at 12 weeks of age were sacrificed by cervical dislocation. Right tibia from each mouse was dissected, the flesh around the bone removed and fixed in 70\% EtOH. Prior to scanning, tibiae were removed from $70 \% \mathrm{EtOH}$ and dried superficially on paper 
tissue, before being wrapped in plastic "cling-film," to prevent drying during scanning, and scanned ex vivo using the Skyscan 1176 (Skyscan, Kontich, Belgium), with X-ray tube operated at $50 \mathrm{kV}$ and $600 \mu \mathrm{A}, 2,000 \mathrm{~ms}$ exposure time, a rotation step of $0.800^{\circ}$, a $1-\mathrm{mm}$ aluminum filter and a voxel size of $9 \mu \mathrm{m}$ within a field of view of $11.5 \mathrm{~mm}$ (width) and $7.8 \mathrm{~mm}$ (height). The slices were then reconstructed using NRecon 1.6.9.4 (Skyscan, Kontich, Belgium). Whole bone analysis was performed on datasets derived from CT scans using BoneJ (Doube et al., 2010) (version 1.4.0) a plugin for ImageJ (Schneider et al., 2012). Following alignment and removal of fibula from the dataset a global bone threshold of 75 Gy level was used to segment bone from non-bone in the images. To determine whether Sost deficiency alters cortical bone architecture, we undertook gross bone morphology analysis. The cross-sectional area (CSA), second moment of area around minor $\left(\mathrm{I}_{\min }\right)$ and major axes $\left(\mathrm{I}_{\max }\right)$ and mean thickness were calculated within BoneJ ("Slice Geometry"). The most proximal and the most distal 10\% portions of tibial length included trabecular bone and thus were not included in the analysis.

\section{Nano-Computed Tomography}

The same tibiofibular junction of the Sost KO and WT mice tibiae scanned previously by micro-CT (2.2.1.1), were re-scanned using a Skyscan 1172 (Skyscan, Kontich, Belgium) X-ray microtomography as described previously (Javaheri et al., 2015). This scanner offers isotropic detail detectability down to $0.5 \mu \mathrm{m}$. The samples were placed in Orthodontic Wax (Kerr, CA, USA) at $200 \mu \mathrm{A}$, $50 \mathrm{kV}$ and, 9,800 $\mathrm{ms}$ exposure time with a $0.25-\mathrm{mm}$ aluminum filter (99.999\% purity, Goodfellow, Huntington, UK), 360 at a rotation step of $0.25^{\circ}$ and a voxel size of $0.6 \mu \mathrm{m}$. Scans were centered at the tibiofibular junction within a field of view of $2.3 \mathrm{~mm}$ (width) and $1.6 \mathrm{~mm}$ (height). Two-frame averaging was used to improve the signal-to-noise ratio. The scan time for each sample was approximately $7 \mathrm{~h}$. Prior to reconstruction, thermal shift in projection images was corrected in NRecon 1.6.9.4 (Skyscan, Kontich, Belgium). 300 slices ( $0.6 \mu \mathrm{m}$ per each slide, total $180 \mu \mathrm{m}$ ) were then reconstructed in NRecon using a ring correction factor of 15, smoothing of 1 and 35\% beam hardening correction. Three 100 consecutive images from the tibiofibular junction were selected from each specimen. The images were loaded in CTAn software (Skyscan, Kontich, Belgium). The major differences between nanoCT and microCT (2.2.1.1) are the voxel size, exposure time, and angular rotation step.

Initially, foreground was segmented from background and a series of noise removal "despeckling" steps performed. Pores smaller than $13 \mu \mathrm{m}^{3}$ and larger than $1,500 \mu \mathrm{m}^{3}$ were assumed to be noise and vascular canals, respectively, and the rest were considered to be lacunae. These limits were based on a previous study using confocal microscopy indicating a size between 28 and $1,713 \mu \mathrm{m}^{3}$ for osteocytes (McCreadie et al., 2004). Previous studies used and reported these volume limits to examine lacunar and canal porosity (Tommasini et al., 2012; Carter et al., 2013; Carriero et al., 2014; Javaheri et al., 2015). Morphometric indices for lacunar and vascular canals were calculated by measuring the $3 \mathrm{D}$ parameters of each discreet object within the volume of interest after segmentation. These indices for lacuna included average lacunar number (N.Lc), average lacunar volume (Lc.Avg.V), total volume (Lc.Tot.V), diameter (Lc.D; calculated with the sphere-fitting method), number of lacunar pores per unit bone volume (N.Lc/Ct.BV), and number of lacunar pores per total volume (N.Lc/Tot.V). For vascular canals, number (Ca.N), total volume (Ca.Tot.V), diameter (Ca.D; calculated with the sphere-fitting method), number of vascular canals per unit bone volume (N.Ca/Ct.BV), and number of vascular canals per total volume (N.Ca/Tot.V) were measured. Shape analysis of the lacunae was conducted utilizing "Analyze Particles" function in BoneJ. Shape parameters were then computed for each ellipsoid based upon the resulting three radii. The best-fit ellipsoid provided lacuna major radius (Lc. $\lambda 1$ ), lacuna intermediate radius (Lc. $\lambda 2$ ), and lacuna minor radius (Lc. $\lambda 3$ ), which correspond to the lacuna's principal axes (i.e., the eigenvalues of the inertial matrix). These values allowed calculation of the degree of lacunar elongation $[\mathrm{Lc} . \mathrm{El}=1-(\mathrm{Lc} . \lambda 2 / \mathrm{Lc} . \lambda 1)]$ and degree of lacunar flatness [Lc.Fl $\geq 1-($ Lc. $\lambda 3 /$ Lc. $\lambda 2)]$ (Javaheri et al., 2015). The composition of the structure was then plotted using a Flinn diagram (Flinn, 1962) showing major: intermediate axis ratio on the $y$-axis and the intermediate: minor axis ratio on the $x$-axis.

\section{Synchrotron NanoCT}

Tibiae from separate groups of male Sost KO and WT mice $(n=6 /$ group) were fixed in $70 \% \mathrm{EtOH}$ and were embedded in wax to prevent sample movement. Tibiofibular junctions were scanned using SR CT at the TOMCAT beamline of the Swiss Light Source at a voxel size of $0.65 \mu \mathrm{m}$. This scanner offers isotropic detail detectability down to $0.37 \mu \mathrm{m}$. For each scan projection, images were acquired over a range of $360^{\circ}$ at a rotation step of $0.12^{\circ}$, a photon energy of $18.5 \mathrm{keV}, 18 \mathrm{~ms}$ exposure, corrected for ring artifacts due to potential scintillator defects and reconstructed based on gridrec algorithm using graphical user interface written in Python/Jython (Marone and Stampanoni, 2012) and developed as a plugin for Fiji (Schindelin et al., 2012). Scans were centered at the tibiofibular junction (maximum outer dimension of $1.5 \mathrm{~mm}$ ) within a field of view of $3.3 \mathrm{~mm}$ (width) and $1.4 \mathrm{~mm}$ (height). Tomographic datasets were typically acquired in 10-15 min. SR CT datasets consisted of a stack of 1,000 reconstructed CT slices, 300 of which were used for morphometric analysis as described previously (see Nano-Computed Tomography).

\section{Statistical Analysis}

Normality and homogeneity of variance were used for all comparisons between Sost KO and WT mice. Violations of normality and homogeneity were not observed.

For gross cortical bone morphology analysis, graphs were plotted using the programming language "R," version 3.1.3 (R Foundation for Statistical Computing, Vienna, Austria; http://www.r-project. org). For this purpose, the functions lattice and grid were used. Two-sample $t$-test was used for comparisons between Sost KO and WT mice. Data are presented as mean \pm SEM and were considered statistically significant when $p<0.05$.

Graphs relating to lacunar and vascular porosity data, obtained from either benchtop nanoCT or synchrotron were generated using GraphPad Prism 6 (GraphPad Software, Inc., San Diego, 
CA, USA). Two-sample $t$-test was used for comparisons between Sost KO and WT mice. Data are presented as mean \pm SEM and were considered statistically significant when $p<0.05$.

\section{RESULTS}

\section{Sost Deficiency Produce Gross Changes in Cortical Bone}

We found that Sost deficiency did not alter tibial length (Table 1) but was a significant determinant of bone CSA (Figure 1B), producing higher bone CSA in Sost KO compared with WT mice along the entire tibia length. Furthermore, we found that Sost deficiency also contributed significantly to cortical thickness (Figures 1A,B), with Sost KO mice exhibiting higher thickness than WT mice. These increases in cortical thickness were accompanied by reduction in medullary cavity area (Figure 1C).

Our data showed that the overall effect of Sost deficiency on $I_{\min }$ was most pronounced distal to the mid-shaft, where significant increases in $\mathrm{I}_{\min }$ were observed (Figure 1D), and that there was a lack of marked alteration in the proximal tibia. $I_{\max }$ was higher 30 and $85 \%$ along the tibia of Sost KO mice (Figure 1D). Predicted tibial resistance to torsion $(\mathrm{J})$ is higher in Sost KO mice in two regions at $\sim 25-35$ and $70-90 \%$ of tibial length (Figure 1E). Moreover, we found that Sost deficiency did not modify tibial ellipticity, a measure of bone shape (Figure 1E).

\section{Sost Deficiency does not Alter Lacunar and Vascular Configurations}

Our data show that Sost deficiency leads to higher total volume (Tot.V) and bone cortical volume (Ct.BV) $(p<0.001$; Table 1). Our morphometric evaluation of the cortical bone at the tibiofibular junction (Figures 2A,B), shows that absolute number of lacunar pores is greater in Sost KO than in WT bones, containing significantly greater total numbers of osteocyte lacunae (N.Lc; $p<0.001$; Figures 2C,D) in images obtained from nanoCT and synchrotron. This greater lacunar number, however, was normalized to control WT mouse levels, when expressed per unit of bone volume (N.Lc/Ct.BV; Figure 2D) as well as total volume including bone, lacuna, and vascular canals volume (N.Lc/Tot.V; Table 1). The average lacunar volume (Lc.Avg.V; Table 1), total lacunar volume occupied by all lacunar pores (Lc.Tot.V; Table 1), and lacunar diameter (Lc.D; Table 1) were not significantly different in datasets of either imaging modalities between Sost KO and WT mice.

Greater absolute non-normalized N.Lc in Sost KO bone was also consistent with measures of greater N.Ca, in which significantly higher vascular canal number (N.Ca; $p<0.001$; Figures 2C,D), were evident in Sost KO bones. This was also normalized to control WT mouse levels when total vascular canal number was expressed per unit bone volume (N.Ca/ Ct.BV; Figure 2D) as well as total volume (N.Ca/Tot.V; Table 1). Furthermore, we found that Sost deficiency did not alter average volume of vascular canals (Ca.Avg.V; Table 1), total volume of all vascular canals (Ca.Tot.V; Table 1), or vascular canal diameter (Ca.D; Table 1). These data indicate that the Sost deficiency produces an elevation in bone volume but does not lead to modification in lacunar and vascular network density in bone.

Analysis of lacunar shape, using Flinn diagram (Flinn, 1962) (Figure 2E) showed that Sost deficiency does not modify osteocyte arrangement and shape. Lacunar elongation and flatness were not significantly altered in tibiofibular junction in Sost KO compared with WT in either imaging modalities. These data showed that Sost deficiency does not lead to divergence in osteocyte shape and organization in mouse tibiae.

\section{DISCUSSION}

Our data are consistent with previous studies (Krishnan et al., 2006; Li et al., 2008; Hassler et al., 2014; Suen et al., 2015; Kamiya

TABLE 1 | Porosity parameters representing lacuna and vascular porosity of male wild-type (WT) and Sost knockout (KO) mice at 12 weeks of age, detailing $t$-test comparisons for significant genotype effect of data obtained from nano-computed tomography (nanoCT) or synchrotron.

\begin{tabular}{|c|c|c|c|c|c|c|}
\hline Morphometric parameters & WT synchrotron & KO synchrotron & $t$-Test & WT CT & KO CT & $t$-Test \\
\hline \multicolumn{7}{|l|}{ Bone parameters } \\
\hline Tibial length (mm) & $17.39 \pm 0.03$ & $17.36 \pm 0.03$ & NS & $17.46 \pm 0.04$ & $17.23 \pm 0.12$ & NS \\
\hline Ct.BV $\left(\mathrm{mm}^{3}\right)$ & $0.146 \pm 0.003$ & $0.205 \pm 0.006$ & $<0.001$ & $0.142 \pm 0.002$ & $0.209 \pm 0.001$ & $<0.001$ \\
\hline Tot.V (bone + canal and lacunar pores) $\left(\mathrm{mm}^{3}\right)$ & $0.151 \pm 0.003$ & $0.211 \pm 0.006$ & $<0.001$ & $0.145 \pm 0.002$ & $0.213 \pm 0.001$ & $<0.001$ \\
\hline \multicolumn{7}{|l|}{ Vascular canal } \\
\hline Ca.Avg.V $\left(\mu \mathrm{m}^{3}\right)$ & $4,529 \pm 870$ & $3,206 \pm 1,201$ & NS & $3,418 \pm 671$ & $2,444 \pm 916$ & NS \\
\hline Ca.Tot.V $\left(\mathrm{mm}^{3}\right)$ & $0.00035 \pm 0.00004$ & $0.00023 \pm 0.00005$ & NS & $0.00021 \pm 0.00008$ & $0.00028 \pm 0.00002$ & NS \\
\hline Ca.D $(\mu \mathrm{m})$ & $9.44 \pm 0.81$ & $7.93 \pm 0.81$ & NS & $8.21 \pm 0.33$ & $7.61 \pm 0.24$ & NS \\
\hline N.Ca/Tot.V (number/mm³) & $443 \pm 68$ & $605 \pm 605$ & NS & $571 \pm 45$ & $691 \pm 242$ & NS \\
\hline \multicolumn{7}{|l|}{ Lacunae } \\
\hline Lc.Avg.V $\left(\mu \mathrm{m}^{3}\right)$ & $396 \pm 46$ & $420 \pm 31$ & NS & $312 \pm 19$ & $288 \pm 40$ & NS \\
\hline Lc.Tot.V $\left(\mathrm{mm}^{3}\right)$ & $0.00581 \pm 0.00002$ & $0.00659 \pm 0.00004$ & NS & $0.00377 \pm 0.00002$ & $0.00428 \pm 0.00001$ & NS \\
\hline Lc.D $(\mu \mathrm{m})$ & $3.82 \pm 0.28$ & $3.39 \pm 0.14$ & NS & $3.13 \pm 0.39$ & $2.9 \pm 0.21$ & NS \\
\hline N.Lc/Tot.V (number/mm³) & $63,189 \pm 863$ & $64,411 \pm 1,240$ & NS & $45,494 \pm 4,170$ & $42,761 \pm 5,741$ & NS \\
\hline
\end{tabular}

Bone parameters included tibial length, cortical bone volume (Ct.BV), and total volume (Tot.V: volumes of bone plus lacunar and canal volumes). For vascular canals, average canal volume (Ca.Avg.V), total canal volume (Ca.Tot.V), canal diameter (Ca.D), and number of vascular canals per total volume (N.Ca/Tot.V) are shown. Morphometric measurements for lacunar pores, include average lacunar volume (LC.Avg.V), total lacunar volume (Lc. Tot.V), lacunar diameter (Lc.D), and number of lacunar pores per total volume (N.LC/Tot.V). WT and Sost KO tibiae scanned at the synchrotron were from a separate batch compared to micro-CT and benchtop nanoCT. Two-sample $t$-test was used to compare means between $W T$ and Sost KO mice. Data represent means \pm SEM with group sizes of $n=6$ for WT and Sost KO mice. 


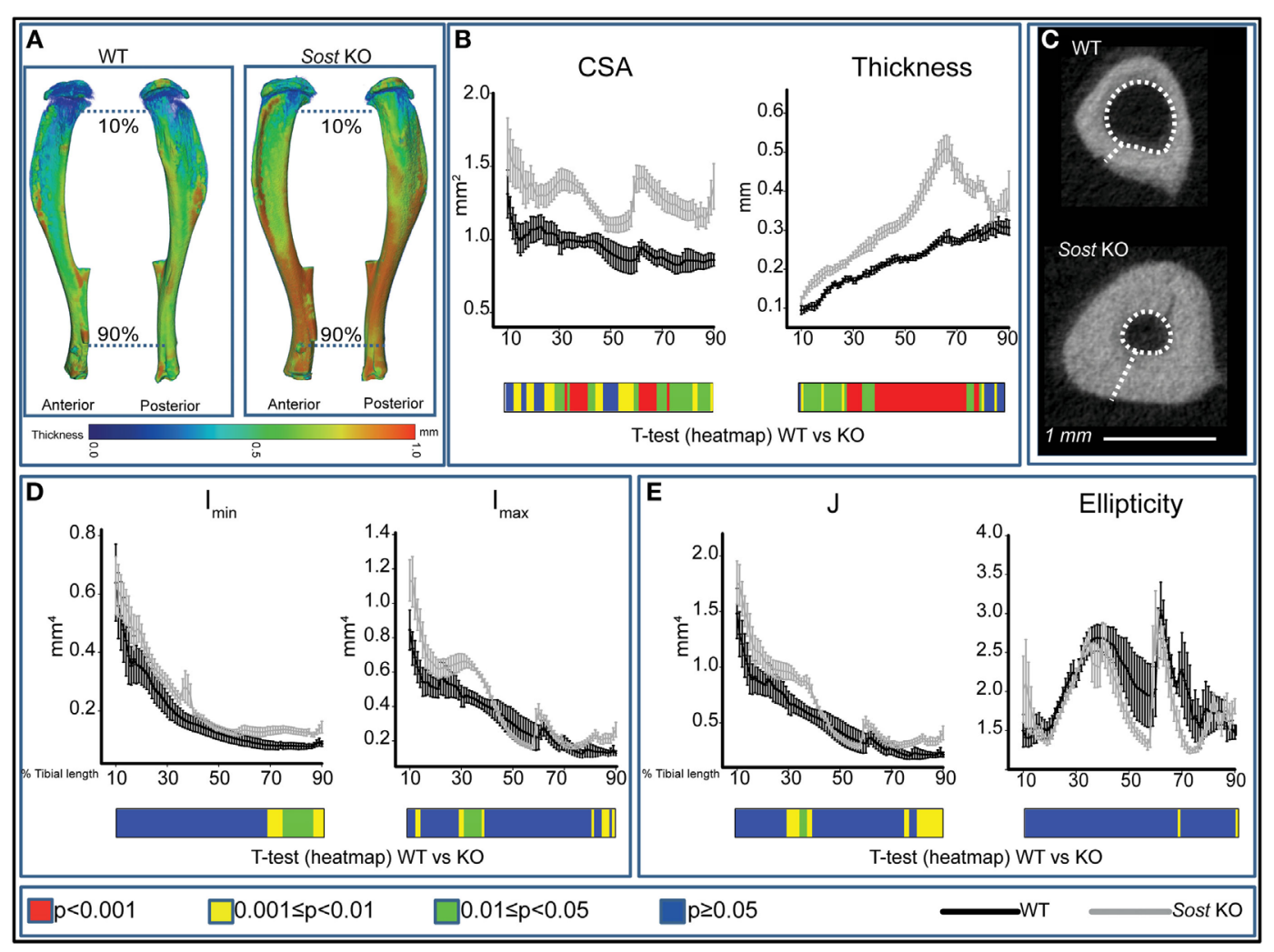

FIGURE 1 | Modification in cortical bone phenotype in Sost knockout (KO) (gray) mice. (A) Representative 3D Micro-CT color-coded images of tibial cortical bone thickness. (B) Bone cross-sectional area (CSA) and mean cortical thickness between 10 and $90 \%$ of total tibial length. (C) Gross macroscopic comparison of microCT images of cortical tibia at the tibiofibular junction for both groups demonstrating a significantly thickened tibial cortex and reduced medullary cavity in Sost KO compared to wild-type (WT) mice. (D) Minimum and maximum second moments of area ( $I_{\min }$ and $I_{\max }$ respectively) and (E) ellipticity and $\mathrm{J}$ (resistance to torsion) between 10 and $90 \%$ of total tibial length of WT and Sost KO mice. Graphical heat map (B,D,E) at the bottom of each graph summarizes statistical differences at specific matched locations along the tibial length, representative of overall effect of Sost deficiency: red $p<0.001$, yellow $0.001 \leq p<0.01$, green $0.01 \leq p<0.05$, and blue $p \geq 0.05$. Two-sample $t$-test was used to compare means between WT and Sost KO mice. Line graphs represent means \pm SEM. Group sizes were $n=6$ for WT and Sost KO mice.

et al., 2016; Qin et al., 2016; Shu et al., 2017) indicating that Sost deficiency leads to significant elevations of cortical bone mass. In addition, we find that these increases are not linked to significant changes in bone shape. Moreover, using two high-resolution imaging modalities, we examined whether the observed elevation of bone mass at macroscopic level in Sost KO mice extends to include differences in the lacunar and vascular composition of cortical bone. In particular, this focuses on the number and morphometric properties of the vascular and osteocyte lacunar cavities within the cortical bone in these mice.

We have previously used benchtop CT to study lacunar and vascular canal porosity using tibiofibular junction as a landmark (Javaheri et al., 2015). Benchtop nanoCT is essentially a micro-CT scanner with small enough focal spot, capable to be used at also sub-micrometer voxel size $(0.6 \mu \mathrm{m} / \mathrm{pixel})$. Herein, we have also employed synchrotron-based nanoCT imaging to compare and confirm suitability of using benchtop-based nanoCT imaging for porosity analysis. With both imaging modalities, the absolute numbers of lacunar and vascular cavities were significantly higher in Sost KO compared with WT mice at tibiofibular junction. This similarity suggests that data obtained from the benchtop CT are comparable, at least in terms of these parameters, to gold standard synchrotron imaging. We could not perform a paired comparison between benchtop and synchrotron nanoCT on the same specimens as bones scanned at the synchrotron were from a different batch.

Our detailed analyses reveal that there were no significant differences in shape, density or organization of lacunae or vascular canal porosity within the cortical bone of Sost KO mice. These data suggest that enhanced properties of bone on a macroscopic scale, specifically in terms of mass in Sostdeficient mice, are not reflected in significant alterations in the geometry and morphometry of lacunar and vascular porosity. The lacunar and vascular canal values per unit volume of bone obtained from both imaging modalities were similar to those reported previously (Carriero et al., 2014; Hemmatian et al., 2017). In addition, values relating to volume from both imaging modalities revealed that Sost deficiency does not alter average and total volume of lacunar or vascular canals. Diameter of lacunar and vascular canals were lower in $\mathrm{KO}$ compared with WT tibiae, but these differences did not reach levels of statistical 


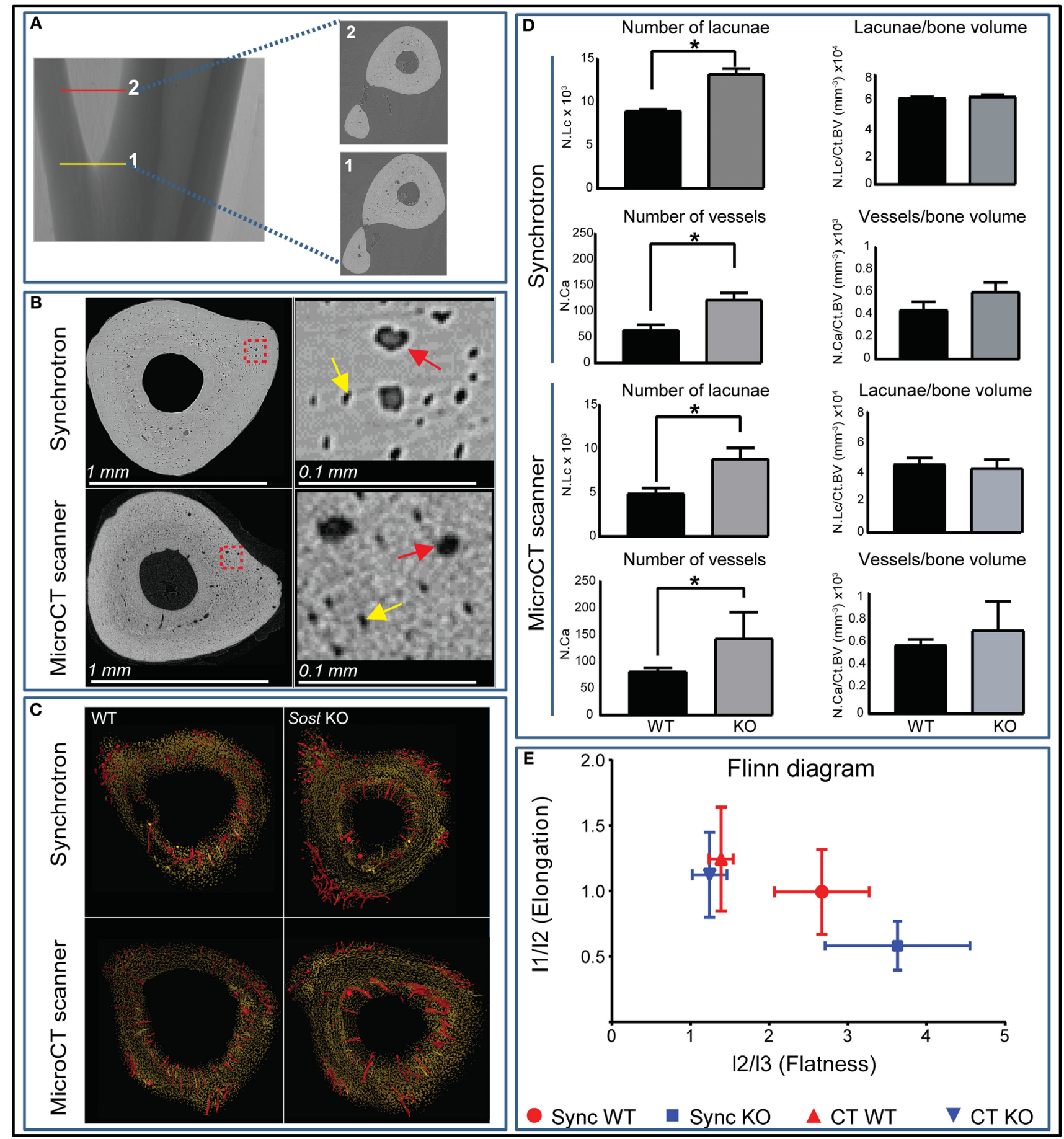

FIGURE 2 | High-resolution nano-computed tomography (nanoCT) and synchrotron-based analysis of the cortex lacunar and vascular porosity at the tibiofibular junction. (A) Three 100 ascending images were selected from point 1 at the tibiofibular junction to point 2 and were used for porosity analysis. (B) Resolving power and gross macroscopic comparison of images obtained from the nanoCT and the synchrotron. Higher magnification quadrants are also shown right side of their respective images for better appreciation. Yellow and red arrows depict selected lacunar and canal pores in both imaging modalities. (C) Surface representation of the lacunar (yellow) and red (vascular canal porosity) segmented from 300 consecutive images from tibiofibular junction of wild-type (WT) and Sost knockout (KO) mice. (D) Number of lacunar pores and vascular canals of WT and Sost KO mice at 12 weeks of age. (E) Flinn diagram displaying lacunar shapes in WT and Sost $\mathrm{KO}$ tibia at tibiofibular junction. The $x$-axis represents lacunar flatness which was calculated by dividing lacunar intermediate radius (I2: length of best-fit ellipsoid's intermediate radius) with lacunar minor radius (I3: length of best-fit ellipsoid's minor radius). The $y$-axis represents lacunar elongation which was calculated by dividing lacunar major radius (11: length of best-fit ellipsoid's major radius) with lacunar intermediate radius (I2: length of best-fit ellipsoid's intermediate radius). WT and Sost KO tibiae scanned at the synchrotron were from a separate batch compared to micro-CT and bench top nanoCT. Two-sample $t$-test was used to compare means between WT and Sost KO mice. Data represent means \pm SEM with group sizes of $n=6$ for WT and Sost KO mice. 
significance. This may explain, minor and not major increases in total volume of lacunar and canal volumes despite having significantly higher absolute number of lacunar and canal cavities. The average volume of lacunar pores was in agreement with those reported previously (Wang et al., 2005; Schneider et al., 2007; Vatsa et al., 2008; Javaheri et al., 2015; Kerckhofs et al., 2016). The average lacunar volume $\left(\sim 360 \mu \mathrm{m}^{3}\right)$ we report in this manuscript is also in agreement with lacunar volume measurements reported by McCreadie et al. (2004) obtained using confocal microscopy.

Furthermore, when the absolute number of both lacunae and vessels are corrected for total and bone volume, the data become not significantly different between Sost KO and WT mice. This implies that the density of lacunae and vascular canals is unaffected by the action of SCLEROSTIN at least at this specific location in male mice at 12 weeks of age. These data suggest that the frequency of osteocyte recruitment from the osteogenic lineage is independent of SCLEROSTIN-mediated control, or alternatively that other factors ensure control of these recruitment processes and that there is, therefore, significant redundancy in this process. The statistically significant increases in bone mass in Sost KO mice implies, however, that this terminal stage of osteoblastic to osteocytic differentiation following entrapment in their own mineralized secretions (Dallas and Bonewald, 2010) occurs in a manner that is Sost-independent. Previous studies reported that the Wnt signaling and SCLEROSTIN regulate bone remodeling (Balemans et al., 2001; Van Wesenbeeck et al., 2003), influence pre-osteoblast differentiation and proliferation (Kato et al., 2002) and osteoblast-to-osteocyte transition in vitro (Atkins et al., 2011). We initially hypothesized that SCLEROSTIN influences the final stage of osteoblast differentiation in bone and expected the values related to osteocyte lacunae per unit bone volume in Sost KO mice to be significantly modified. As this does not appear to be the case, our data offer weight to the argument that any interaction between SCLEROSTIN and the maturation pathway occurs at an earlier stage in osteoblast cell lineage commitment. Similar arguments are also applicable to the role of SCLEROSTIN in vascular recruitment to bone. Further work should thus concentrate on identifying the exact stage or stages that are significantly influenced by the presence or absence of SCLEROSTIN.

\section{REFERENCES}

Armstrong, V. J., Muzylak, M., Sunters, A., Zaman, G., Saxon, L. K., Price, J. S., et al. (2007). Wnt/beta-catenin signaling is a component of osteoblastic bone cell early responses to load-bearing and requires estrogen receptor alpha. J. Biol. Chem. 282, 20715-20727. doi:10.1074/jbc.M703224200

Atkins, G. J., Rowe, P. S., Lim, H. P., Welldon, K. J., Ormsby, R., Wijenayaka, A. R., et al. (2011). Sclerostin is a locally acting regulator of late-osteoblast/preosteocyte differentiation and regulates mineralization through a MEPE-ASARMdependent mechanism. J. Bone Miner. Res. 26, 1425-1436. doi:10.1002/ jbmr.345

Babij, P., Zhao, W., Small, C., Kharode, Y., Yaworsky, P. J., Bouxsein, M. L., et al. (2003). High bone mass in mice expressing a mutant LRP5 gene. J. Bone Miner. Res. 18, 960-974. doi:10.1359/jbmr.2003.18.6.960

Balemans, W., Cleiren, E., Siebers, U., Horst, J., and Van Hul, W. (2005). A generalized skeletal hyperostosis in two siblings caused by a novel mutation in the SOST gene. Bone 36, 943-947. doi:10.1016/j.bone.2005.02.019
There are several limitations to our study. The number of samples in our study are six per group and variation in our data, specifically the KO group (Table 1 and Figure 2D), might have influenced our findings. In this context, it is worth noting that murine bones lack Haversian canals and that it is possible that the role of SCLEROSTIN, in species where such canals comprise a significant volume of the cortex (humans), may differ (Perilli et al., 2015). In addition, we find that canal and lacunar porosity volumes recorded by benchtop CT are smaller than those revealed by synchrotron, by 40 and 35\%, respectively. It is possible that despite the similar diameter of these pores with the two CT systems, there could be some artificially broken canals and hence smaller volume measured by the benchtop CT (Palacio-Mancheno et al., 2014).

In conclusion, our data show that Sost deficiency in mice, despite having a significant influence on the physical and structural properties of the whole bone does not modify lacunar and vascular canal porosities. Thus, SCLEROSTIN's negative modulatory role on bone formation does not likely extend to a control over the terminal phase in osteocyte recruitment nor to any modification in bone's vascularization in mice.

\section{ETHICS STATEMENT}

This study was carried out in accordance with the recommendations and approval of ethics committee of the Royal Veterinary College (London, UK). All procedures complied with the UK Animals (Scientifc Procedures) Act 1986.

\section{AUTHOR CONTRIBUTIONS}

Conceived and designed the experiments: CC, AP, and BJ. Performed the experiments: BJ, JN, and AG. Analyzed the data: $\mathrm{HM}$ and BJ. Contributed reagents/materials/analysis tools: $\mathrm{KS}$, CC, PL, and AP. Wrote the paper: BJ and AP.

\section{FUNDING}

This study was supported by funding from the BBSRC BB/ I014608/1, Arthritis Research UK 20581, and 20039 and The Society for Endocrinology Early Career Grant.

Balemans, W., Ebeling, M., Patel, N., Van Hul, E., Olson, P., Dioszegi, M., et al. (2001). Increased bone density in sclerosteosis is due to the deficiency of a novel secreted protein (SOST). Hum. Mol. Genet. 10, 537-543. doi:10.1093/hmg/10.5.537

Bélanger, L. F. (1969). Osteocytic osteolysis. Calcif. Tissue Int. 4, 1-12. doi:10.1007/ BF02279101

Bennell, K. L., Khan, K. M., Warmington, S., Forwood, M. R., Coleman, B. D., Bennett, M. B., et al. (2002). Age does not influence the bone response to treadmill exercise in female rats. Med. Sci. Sports Exerc. 34, 1958-1965. doi:10.1097/ 00005768-200212000-00015

Bodine, P. V., Zhao, W., Kharode, Y. P., Bex, F. J., Lambert, A.-J., Goad, M. B., et al. (2004). The Wnt antagonist secreted frizzled-related protein-1 is a negative regulator of trabecular bone formation in adult mice. Mol. Endocrinol. 18, 1222-1237. doi:10.1210/me.2003-0498

Bonewald, L. F., and Johnson, M. L. (2008). Osteocytes, mechanosensing and Wnt signaling. Bone 42, 606-615. doi:10.1016/j.bone.2007.12.224

Burger, E. H., and Klein-Nulend, J. (1999). Mechanotransduction in bone - role of the lacuno-canalicular network. FASEB J. 13(Suppl.), S101-S112. 
Cameron, D. A., Paschall, H. A., and Robinson, R. A. (1967). Changes in the fine structure of bone cells after the administration of parathyroid extract. J. Cell Biol. 33, 1-14. doi:10.1083/jcb.33.1.1

Carriero, A., Doube, M., Vogt, M., Busse, B., Zustin, J., Levchuk, A., et al. (2014). Altered lacunar and vascular porosity in osteogenesis imperfecta mouse bone as revealed by synchrotron tomography contributes to bone fragility. Bone 61, 116-124. doi:10.1016/j.bone.2013.12.020

Carter, Y., Thomas, C. D. L., Clement, J. G., Peele, A. G., Hannah, K., and Cooper, D. M. (2013). Variation in osteocyte lacunar morphology and density in the human femur - a synchrotron radiation micro-CT study. Bone 52, 126-132. doi:10.1016/j.bone.2012.09.010

Choi, I. H., Ahn, J. H., Chung, C. Y., and Cho, T. J. (2000). Vascular proliferation and blood supply during distraction osteogenesis: a scanning electron microscopic observation. J. Orthop. Res. 18, 698-705. doi:10.1002/jor.1100180504

Cooper, D. M., Matyas, J. R., Katzenberg, M. A., and Hallgrimsson, B. (2004). Comparison of microcomputed tomographic and microradiographic measurements of cortical bone porosity. Calcif. Tissue Int. 74, 437-447. doi:10.1007/ s00223-003-0071-z

Dallas, S. L., and Bonewald, L. F. (2010). Dynamics of the transition from osteoblast to osteocyte. Ann. N. Y. Acad. Sci. 1192, 437-443. doi:10.1111/j.1749-6632. 2009.05246.x

Dong, P., Pacureanu, A., Zuluaga, M. A., Olivier, C., Frouin, F., Grimal, Q., et al. (eds) (2013). "A new quantitative approach for estimating bone cell connections from nano-CT images," in Engineering in Medicine and Biology Society (EMBC), 2013 35th Annual International Conference of the IEEE, Osaka, Japan: (IEEE).

Doube, M., Klosowski, M. M., Arganda-Carreras, I., Cordelieres, F. P., Dougherty, R. P., Jackson, J. S., et al. (2010). BoneJ: free and extensible bone image analysis in ImageJ. Bone 47, 1076-1079. doi:10.1016/j.bone.2010.08.023

Flinn, D. (1962). On folding during three-dimensional progressive deformation. Q. J. Geol. Soc. 118, 385-428. doi:10.1144/gsjgs.118.1.0385

Franz-Odendaal, T. A., Hall, B. K., and Witten, P. E. (2006). Buried alive: how osteoblasts become osteocytes. Dev. Dyn. 235, 176-190. doi:10.1002/dvdy.20603

Glass, D. A. II, Bialek, P., Ahn, J. D., Starbuck, M., Patel, M. S., Clevers, H., et al. (2005). Canonical Wnt signaling in differentiated osteoblasts controls osteoclast differentiation. Dev. Cell 8, 751-764. doi:10.1016/j.devcel.2005.02.017

Han, Y.,Cowin,S.C.,Schaffler,M.B., andWeinbaum,S.(2004). Mechanotransduction and strain amplification in osteocyte cell processes. Proc. Natl. Acad. Sci. U.S.A. 101, 16689-16694. doi:10.1073/pnas.0407429101

Hassler, N., Roschger, A., Gamsjaeger, S., Kramer, I., Lueger, S., van Lierop, A., et al. (2014). Sclerostin deficiency is linked to altered bone composition. J. Bone Miner. Res. 29, 2144-2151. doi:10.1002/jbmr.2259

Hemmatian, H., Laurent, M. R., Ghazanfari, S., Vanderschueren, D., Bakker, A. D., Klein-Nulend, J., et al. (2017). Accuracy and reproducibility of mouse cortical bone microporosity as quantified by desktop microcomputed tomography. PLoS ONE 12:e0182996. doi:10.1371/journal.pone.0182996

Holmen, S. L., Zylstra, C. R., Mukherjee, A., Sigler, R. E., Faugere, M. C., Bouxsein, M. L., et al. (2005). Essential role of beta-catenin in postnatal bone acquisition. J. Biol. Chem. 280, 21162-21168. doi:10.1074/jbc.M501900200

Howell, J. (1917). An experimental study of the effect of stress and strain on bone development. Anat. Rec. 13, 233-252. doi:10.1002/ar.1090130502

Järvinen, T. L., Pajamäki, I., Sievänen, H., Vuohelainen, T., Tuukkanen, J., Järvinen, M., et al. (2003). Femoral neck response to exercise and subsequent deconditioning in young and adult rats. J. Bone Miner. Res. 18, 1292-1299. doi:10.1359/jbmr.2003.18.7.1292

Javaheri, B., Carriero, A., Staines, K. A., Chang, Y. M., Houston, D. A., Oldknow, K. J., et al. (2015). Phosphol deficiency transiently modifies bone architecture yet produces consistent modification in osteocyte differentiation and vascular porosity with ageing. Bone 81, 277-291. doi:10.1016/j.bone.2015.07.035

Javaheri, B., Stern, A. R., Lara, N., Dallas, M., Zhao, H., Liu, Y., et al. (2014). Deletion of a single beta-catenin allele in osteocytes abolishes the bone anabolic response to loading. J. Bone Miner. Res. 29, 705-715. doi:10.1002/jbmr.2064

Kamiya, N., Shuxian, L., Yamaguchi, R., Phipps, M., Aruwajoye, O., Adapala, N. S., et al. (2016). Targeted disruption of BMP signaling through type IA receptor (BMPR1A) in osteocyte suppresses SOST and RANKL, leading to dramatic increase in bone mass, bone mineral density and mechanical strength. Bone 91, 53-63. doi:10.1016/j.bone.2016.07.002

Kato, M., Patel, M. S., Levasseur, R., Lobov, I., Chang, B. H., Glass, D. A. II, et al. (2002). Cbfal-independent decrease in osteoblast proliferation, osteopenia, and persistent embryonic eye vascularization in mice deficient in Lrp5, a Wnt coreceptor. J. Cell Biol. 157, 303-314. doi:10.1083/jcb.200201089

Kerckhofs, G., Durand, M., Vangoitsenhoven, R., Marin, C., Van Der Schueren, B., Carmeliet, G., et al. (2016). Changes in bone macro- and microstructure in diabetic obese mice revealed by high resolution microfocus X-ray computed tomography. Sci. Rep. 6, 35517. doi:10.1038/srep35517

Kerschnitzki, M., Kollmannsberger, P., Burghammer, M., Duda, G. N., Weinkamer, R., Wagermaier, W., et al. (2013). Architecture of the osteocyte network correlates with bone material quality. J. Bone Miner. Res. 28, 1837-1845. doi:10.1002/ jbmr.1927

Krishnan, V., Bryant, H. U., and Macdougald, O. A. (2006). Regulation of bone mass by Wnt signaling. J. Clin. Invest. 116, 1202-1209. doi:10.1172/JCI28551

Leppänen, O. V., Sievänen, H., Jokihaara, J., Pajamäki, I., Kannus, P., and Järvinen, T. L. (2008). Pathogenesis of age-related osteoporosis: impaired mechano-responsiveness of bone is not the culprit. PLOS ONE 3:e2540. doi:10.1371/ journal.pone.0002540

Li, X., Ominsky, M. S., Niu, Q. T., Sun, N., Daugherty, B., D’Agostin, D., et al. (2008). Targeted deletion of the sclerostin gene in mice results in increased bone formation and bone strength. J. Bone Miner. Res. 23, 860-869. doi:10.1359/ jbmr.080216

Ma, Y.-L., Dai, R.-C., Sheng, Z.-F., Jin, Y., Zhang, Y.-H., Fang, L.-N., et al. (2008). Quantitative associations between osteocyte density and biomechanics, microcrack and microstructure in OVX rats vertebral trabeculae. J. Biomech. 41, 1324-1332. doi:10.1016/j.jbiomech.2008.01.017

Mader, K. S., Schneider, P., Müller, R., and Stampanoni, M. (2013). A quantitative framework for the $3 \mathrm{D}$ characterization of the osteocyte lacunar system. Bone 57, 142-154. doi:10.1016/j.bone.2013.06.026

Marone, F., and Stampanoni, M. (2012). Regridding reconstruction algorithm for real-time tomographic imaging. J. Synchrotron. Radiat. 19, 1029-1037. doi:10.1107/S0909049512032864

Matsumoto, T., Yoshino, M., Asano, T., Uesugi, K., Todoh, M., and Tanaka, M. (2006). Monochromatic synchrotron radiation muCT reveals disuse-mediated canal network rarefaction in cortical bone of growing rat tibiae. J. Appl. Physiol. 100, 274-280. doi:10.1152/japplphysiol.00495.2005

McCreadie, B. R., Hollister, S. J., Schaffler, M. B., and Goldstein, S. A. (2004). Osteocyte lacuna size and shape in women with and without osteoporotic fracture. J. Biomech. 37, 563-572. doi:10.1016/S0021-9290(03)00287-2

Mullender, M. G., Tan, S. D., Vico, L., Alexandre, C., and Klein-Nulend, J. (2005). Differences in osteocyte density and bone histomorphometry between men and women and between healthy and osteoporotic subjects. Calcif. Tissue Int. 77, 291-296. doi:10.1007/s00223-005-0043-6

Oranger, A., Brunetti, G., Colaianni, G., Tamma, R., Carbone, C., Lippo, L., et al. (2017). Sclerostin stimulates angiogenesis in human endothelial cells. Bone 101, 26-36. doi:10.1016/j.bone.2017.03.001

Pacureanu, A., Langer, M., Boller, E., Tafforeau, P., and Peyrin, F. (2012). Nanoscale imaging of the bone cell network with synchrotron X-ray tomography: optimization of acquisition setup. Med. Phys. 39, 2229-2238. doi:10.1118/1. 3697525

Palacio-Mancheno, P. E., Larriera, A. I., Doty, S. B., Cardoso, L., and Fritton, S. P. (2014). 3D assessment of cortical bone porosity and tissue mineral density using high-resolution microCT: effects of resolution and threshold method. J. Bone Miner. Res. 29, 142-150. doi:10.1002/jbmr.2012

Perilli, E., Bala, Y., Zebaze, R., Reynolds, K. J., and Seeman, E. (2015). Regional heterogeneity in the configuration of the intracortical canals of the femoral shaft. Calcif. Tissue Int. 97, 327-335. doi:10.1007/s00223-015-0014-5

Prideaux, M., Loveridge, N., Pitsillides, A. A., and Farquharson, C. (2012). Extracellular matrix mineralization promotes E11/gp38 glycoprotein expression and drives osteocytic differentiation. PLOS ONE 7:e36786. doi:10.1371/ journal.pone.0036786

Qin, W., Zhao, W., Li, X., Peng, Y., Harlow, L. M., Li, J., et al. (2016). Mice with sclerostin gene deletion are resistant to the severe sublesional bone loss induced by spinal cord injury. Osteoporos. Int. 27, 3627-3636. doi:10.1007/s00198016-3700-x

Qing, H., and Bonewald, L. F. (2009). Osteocyte remodeling of the perilacunar and pericanalicular matrix. Int. J. Oral Sci. 1, 59. doi:10.4248/ijos.09019

Qiu, S., Rao, D. S., Palnitkar, S., and Parfitt, A. M. (2003). Reduced iliac cancellous osteocyte density in patients with osteoporotic vertebral fracture. J. Bone Miner. Res. 18, 1657-1663. doi:10.1359/jbmr.2003.18.9.1657 
Raab, D. M., Crenshaw, T. D., Kimmel, D. B., and Smith, E. L. (1991). A histomorphometric study of cortical bone activity during increased weight-bearing exercise. J. Bone Miner. Res. 6, 741-749. doi:10.1002/jbmr.5650060712

Reya, T., and Clevers, H. (2005). Wnt signalling in stem cells and cancer. Nature 434, 843-850. doi:10.1038/nature03319

Robling, A. G., and Turner, C. H. (2002). Mechanotransduction in bone: genetic effects on mechanosensitivity in mice. Bone 31, 562-569. doi:10.1016/ S8756-3282(02)00871-2

Rubin, C. T. (1984). Skeletal strain and the functional significance of bone architecture. Calcif. Tissue Int. 36, S11-S18. doi:10.1007/BF02406128

Schindelin, J., Arganda-Carreras, I., Frise, E., Kaynig, V., Longair, M., Pietzsch, T., et al. (2012). Fiji: an open-source platform for biological-image analysis. Nat. Methods 9, 676-682. doi:10.1038/nmeth.2019

Schneider, C. A., Rasband, W. S., and Eliceiri, K. W. (2012). NIH Image to ImageJ: 25 years of image analysis. Nat. Methods 9, 671. doi:10.1038/nmeth.2089

Schneider, P., Krucker, T., Meyer, E., Ulmann-Schuler, A., Weber, B., Stampanoni, M., et al. (2009). Simultaneous 3D visualization and quantification of murine bone and bone vasculature using micro-computed tomography and vascular replica. Microsc. Res. Tech. 72, 690-701. doi:10.1002/jemt.20720

Schneider, P., Meier, M., Wepf, R., and Muller, R. (2010). Towards quantitative 3D imaging of the osteocyte lacuno-canalicular network. Bone 47, 848-858. doi:10.1016/j.bone.2010.07.026

Schneider, P., Stauber, M., Voide, R., Stampanoni, M., Donahue, L. R., and Müller, R. (2007). Ultrastructural properties in cortical bone vary greatly in two inbred strains of mice as assessed by synchrotron light based micro- and nano-CT. J. Bone Miner. Res. 22, 1557-1570. doi:10.1359/jbmr.070703

Shu, R., Ai, D., Bai, D., Song, J., Zhao, M., and Han, X. (2017). The effects of SOST on implant osseointegration in ovariectomy osteoporotic mice. Arch. Oral Biol. 74, 82-91. doi:10.1016/j.archoralbio.2016.11.012

Skedros, J. G., Grunander, T. R., and Hamrick, M. W. (2005). Spatial distribution of osteocyte lacunae in equine radii and third metacarpals: considerations for cellular communication, microdamage detection and metabolism. Cells Tissues Organs 180, 215-236. doi:10.1159/000088938

Steinberg, M. E., and Trueta, J. (1981). Effects of activity on bone growth and development in the rat. Clin. Orthop. Relat. Res. 156, 52-60.

Suen, P. K., Zhu, T. Y., Chow, D. H., Huang, L., Zheng, L. Z., and Qin, L. (2015). Sclerostin antibody treatment increases bone formation, bone mass, and bone strength of intact bones in adult male rats. Sci. Rep. 5, 15632. doi:10.1038/ srep 15632
Tommasini, S. M., Trinward, A., Acerbo, A. S., De Carlo, F., Miller, L. M., and Judex, S. (2012). Changes in intracortical microporosities induced by pharmaceutical treatment of osteoporosis as detected by high resolution micro-CT. Bone 50, 596-604. doi:10.1016/j.bone.2011.12.012

Van Wesenbeeck, L., Cleiren, E., Gram, J., Beals, R. K., Benichou, O., Scopelliti, D., et al. (2003). Six novel missense mutations in the LDL receptor-related protein 5 (LRP5) gene in different conditions with an increased bone density. Am. J. Hum. Genet. 72, 763-771. doi:10.1086/368277

Van Wesenbeeck, L., Odgren, P. R., MacKay, C. A., D’Angelo, M., Safadi, F. F., Popoff, S. N., et al. (2002). The osteopetrotic mutation toothless (tl) is a loss-offunction frameshift mutation in the rat Csfl gene: evidence of a crucial role for CSF-1 in osteoclastogenesis and endochondral ossification. Proc. Natl. Acad. Sci. U.S.A. 99, 14303-14308. doi:10.1073/pnas.202332999

Vatsa, A., Breuls, R. G., Semeins, C. M., Salmon, P. L., Smit, T. H., and KleinNulend, J. (2008). Osteocyte morphology in fibula and calvaria-is there a role for mechanosensing? Bone 43, 452-458. doi:10.1016/j.bone.2008.01.030

Verborgt, O., Gibson, G. J., and Schaffler, M. B. (2000). Loss of osteocyte integrity in association with microdamage and bone remodeling after fatigue in vivo. J. Bone Miner. Res. 15, 60-67. doi:10.1359/jbmr.2000.15.1.60

Wang, L., Wang, Y., Han, Y., Henderson, S. C., Majeska, R. J., Weinbaum, S., et al. (2005). In situ measurement of solute transport in the bone lacunarcanalicular system. Proc. Natl. Acad. Sci. U.S.A. 102, 11911-11916. doi:10.1073/ pnas.0505193102

Webster, D. J., Schneider, P., Dallas, S. L., and Müller, R. (2013). Studying osteocytes within their environment. Bone 54, 285-295. doi:10.1016/j.bone.2013.01.004

Weivoda, M. M., Youssef, S. J., and Oursler, M. J. (2017). Sclerostin expression and functions beyond the osteocyte. Bone 96, 45-50. doi:10.1016/j.bone.2016.11.024

Conflict of Interest Statement: The authors declare that the research was conducted in the absence of any commercial or financial relationships that could be construed as a potential conflict of interest.

Copyright (C) 2017 Mosey, Núñez, Goring, Clarkin, Staines, Lee, Pitsillides and Javaheri. This is an open-access article distributed under the terms of the Creative Commons Attribution License (CC BY). The use, distribution or reproduction in other forums is permitted, provided the original author(s) or licensor are credited and that the original publication in this journal is cited, in accordance with accepted academic practice. No use, distribution or reproduction is permitted which does not comply with these terms. 